\title{
WIRELESS ENERGY TRANSFER USING MICROSTRIP ANTENNA
}

\author{
${ }^{1}$ Leong Kah Meng, ${ }^{1}$ Tan Tian Swee, ${ }^{1}$ Lum Kin Yun and ${ }^{2}$ Johari Kassim \\ ${ }^{1}$ Medical Implant Technology Group (MediTEG), IJN-UTM Cardiovascular Engineering Center, \\ Material Manufacturing Research Alliance (MMRA), \\ Department of Biotechnology and Medical Engineering, \\ Faculty of Biosciences and Medical Engineering (FBME), Universiti Teknologi Malaysia (UTM), Malaysia \\ ${ }^{2}$ Electronic and Computer Engineering (ECE), Faculty of Electrical Engineering, \\ Universiti Teknologi Malaysia, 81310, Skudai, Johor, Malaysia
}

Received 2013-07-12; Revised 2013-09-27; Accepted 2013-12-20

\begin{abstract}
This study presents a concept on wireless energy transmission using microstrip antenna pairs. Microstrip antenna is chosen in its implementation in wireless energy transfer application primarily due to its characteristics: Its ease of analysis, fabrication and their attractive radiation characteristics. The outcome of this research is the fabrication of two microstrip antennas with resonant frequency of $1.94 \mathrm{GHz}$ and $2.5 \mathrm{GHz}$ respectively. The performance and the power gain for each of microstrip antennas which act as the transmitter and receiver respectively were evaluated within certain distance. The limitations of the experiment as well as the possible solutions in increasing system efficiency are being discussed. Experiment shows that the microstrip antenna with a lower resonant frequency performs better in long distance wireless energy transmission.
\end{abstract}

Keywords: Microstrip Antenna, Wireless Energy Transfer

\section{INTRODUCTION}

Nowadays, the usage of electronic devices is increasing exponentially. Electronic devices like smart phones, laptop, iPad and iPod are gradually becoming a need for everyone. All these electronic devices do need a charger. Due to the rapid run out of battery, it is very inconvenience to bring along the charger while having a longer period of roaming. The inevitable weakness of today's typical charger is the power cord. It is rather messy and does limit the mobility in powering the devices (Yu et al., 2011). Therefore, a wireless charger is needed to solve this problem (Lin, 2011).

Some current research on dealing with this issue is like following. Chang and Liao (2010) the method of develop a rectenna using high gain franklin array was used as the receiver antenna. The resonant frequency used is 2.4 GHz. The disadvantage of using Franklin array is that we need to consider the configuration of the array and is complicated when fabricating the antenna. Chang and Liao (2010) total 8 Franklin array elements and total 32 patch elements were used in Franklin array antenna.

Others than using antenna and array, (Peng et al., 2011) use a different way which is using electromagnetic and resonance magnetic coupling method. This concept was based on the interaction of two resonant objects which is capable to maximize the energy transfer. The system is supplied energy to oscillate at its resonant frequency. The limitation of using this method was the range of the transmitter and receiver can only perform in a very short distance.

Yoon and Ling (2012), the design electrically small, Folded Cylindrical Helix $(\mathrm{FCH})$ antennas had been implement and discuss. The resonant frequency for the antenna to operate was $200 \mathrm{MHz}$. This antenna was Corresponding Author: Tan Tian Swee Medical Implant Technology Group (MediTEG), IJN-UTM Cardiovascular Engineering Center, Material Manufacturing Research Alliance (MMRA), Department of Biotechnology and Medical Engineering, Faculty of Biosciences and Medical Engineering (FBME), Universiti Teknologi Malaysia (UTM), Malaysia 
design for long range application therefore a lower resoant frequency was used. The diameter and height of the antenna are chosen to be approximately equal and are confined to a maximum dimension of $10.5 \mathrm{~cm}$. The number of turns (1.25-turn) and the number of arms (4 arms) are chosen. One of weakness for this technique was multiple transmitter are required ion order to achieve a stable power transfer efficiency (PTE) region.

Another method was using Two Folded Shorted Patch Antennas (FSPA) for outdoor RF energy harvesting in powering wireless soil sensor network (Sim et al., 2010). The technique used was RF energy radiated from a controlled transmitter is capture by a receiver antenna which attached to each wireless sensor node. The energy received was then converted to DC voltage through power conversion circuit in order to power up the sensor. The antenna consist a large ground plane size which make it not practical for intended application.

When we talk about antennas, we cannot skip to mention about the Friis transmission equation. The Friis transmission equation is used in telecommunications engineering, it enable us to calculate the power received under idealized conditions when given another antenna some distance away transmitting a known amount of power. We can increase the transmitting energy or shorter the distance between transmitter antenna and receiver antenna in order to power up receive power (Casanova et al., 2009).

The objective of this project is to study the characteristic of microstrip antenna and design an antenna pairs used for power transmission and receiving purpose. Common used frequencies were being selected and become the research interest in this project.

Microstrip antenna transmitter and receiver are needed to implement to solve all the problems that cause inconvenience to human being. In this study, resonant frequency $1.9 \mathrm{GHz}$ to $2.45 \mathrm{GHz}$ will be selected to implement the project. Two pairs small size microstrip antenna with resonant frequency of $1.9 \mathrm{GHz}$ and 2.45 $\mathrm{GHz}$ will be designed and implemented (Heikkinen and Kivikoski, 2001). The transmitting power was 10 $\mathrm{mW}$. We will further discuss the method and designing process of the microstrip antenna for transmitting energy.

\section{MATERIALS AND METHODS}

\subsection{Methodology}

In order to design an antenna which can operate in the desire frequency, AWR Design Environment is chosen to be the design platform for the microstrip antenna for this project. Difference sizes and shapes of microstrip antenna will operate at different resonant frequency.

The mismatch of the resonant frequency will result in a drop in performance. On the other hand, impedance matching does play an important role in energy transmission two port networks. So, the matching of the impedance between the system and the antenna is equally important. This will affect the performance of the antennas. Once you done your designing procedure you can simulate and see the frequency response of the antenna. Repeat the designing steps until the antenna get the desire resonant frequency.

After checking all the waveform and the frequency and make sure the antenna meets the desired specification, then can move to next stage which is fabrication of microstrip antenna. Before going to the fabrication laboratory, another step is needed which is drawing the correct size of microstrip antenna using the AutoCAD. The design in AutoCAD will be printed on a transparency paper. Before put into UV exposure machine, make sure that the transparency paper must cooperate with negative dry film photo resist sheets stick tight to PCB. This UV exposure method usually use in fabrication of antenna due to the antenna required precise fabrication in order to achieve its desired designed frequency. So the normal ironing or laminated methods on PCB must be avoided because it will easily expand the copper in the PCB. The expanded part of the copper will cause the difference between the simulation part which done before so it will gradually affect the performance of the antenna. After the UV exposure process then can move to etching process. When all the process is finish then the microstrip antenna can be said as fabricated successfully. Network analyzer is used to evaluate the frequency response of the fabricated antenna. Every antenna which successfully fabricated must be tested using the network analyzer in order to verify the performance of the antenna happen.

\subsection{Size of Antenna}

Different size of antenna will have different resonant frequency (Goh et al., 2012). Figure 1 show the comparison between the antennas consists of lower resonant frequency $(1.9 \mathrm{GHz})$ and the higher frequency $(2.45 \mathrm{GHz})$ which design using AWR Design Environment.

From the Fig. 1 we can see that the comparison within the size from two different resonant frequency of fabricated microstrip antennas. The size for the microstrip antenna with resonant frequency equal to $1.9 \mathrm{GHz}$ is larger than microstrip antenna with resonant frequency equal to 2.45 GHz. By increasing the size of microstrip antenna can lower down the resonant frequency of microstrip antenna. 

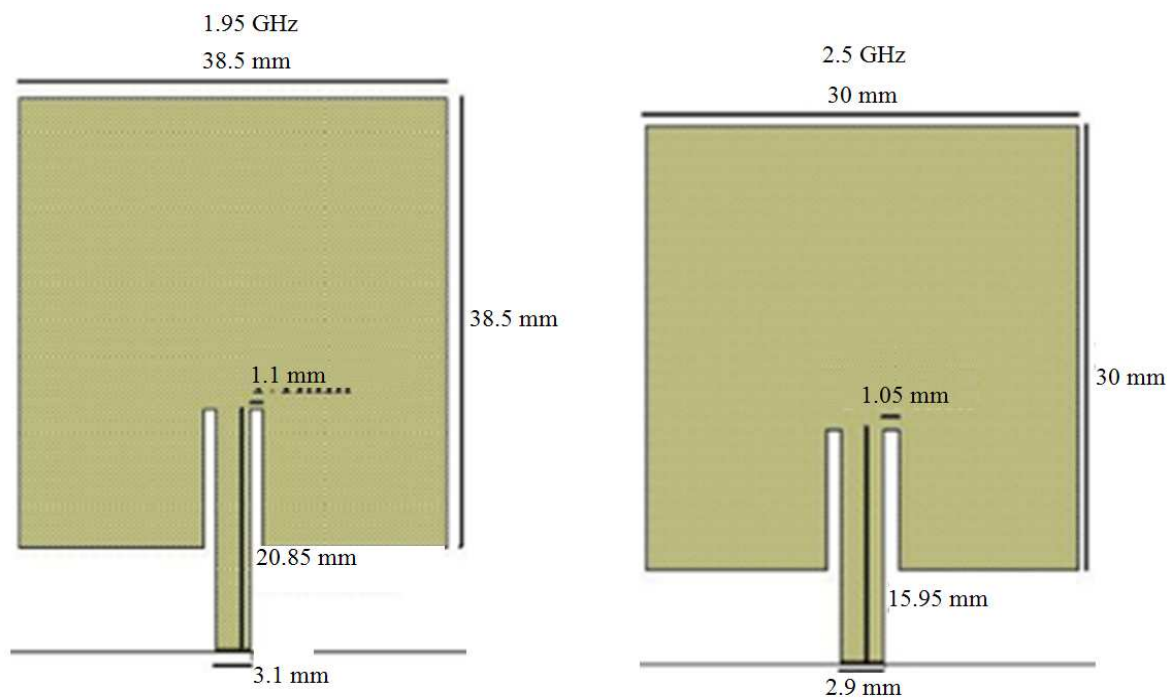

Fig. 1. Microstrip antenna design using AWR design environment

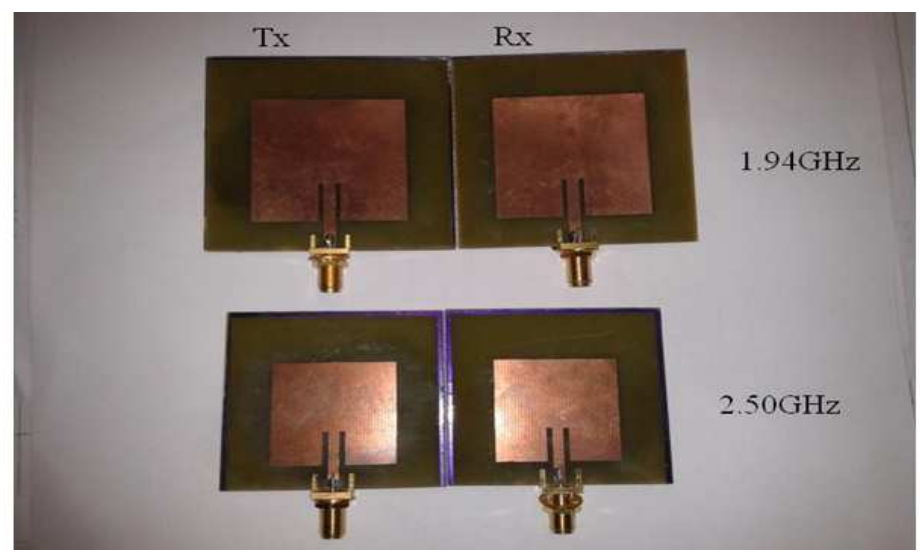

Fig. 2. Fabricated microstrip antennas

In order to get the best resonant frequency for the microstrip antenna, we can do more simulation by using AWR Design Environment software.

From Fig. 2 we can seen that larger size antenna is corresponding to the resonant frequency of $1.94 \mathrm{GHz}$ while smaller size antenna corresponding to the resonant frequency of $2.5 \mathrm{GHz}$.

\subsection{Frequency Response of Antenna}

Figure 3 and 4 shows the frequency response graph of microstrip antenna resonant frequency $2.45 \mathrm{GHz}$ and $1.9 \mathrm{GHz}$ which is simulated using AWR Design Environment.

Figure 5-8 shows the operating frequency response graphs for the fabricated microstrip antennas. They were evaluated using network analyzer. The actual result showed some deviation in frequency as compared to the simulation result. The resonant frequency is $2.5 \mathrm{GHz}$ for Fig. 5 and 6. Meanwhile, it is showing a frequency of $1.94 \mathrm{GHz}$ for Fig. 7 and 8.

\subsection{Measurement}

Sweep values of distance were performed starting 3 $\mathrm{cm}$ to $30 \mathrm{~cm}$ during the measurement process. The reading is taken repeatedly up to two times in order to get the average reading of the output. This is because the high frequency transmission might be affected by noise and other outside frequency disturbance. In order to carry out the measurement Vaunix Lab Bricks and MiniCircuit USB Power Meter were used in this project. 


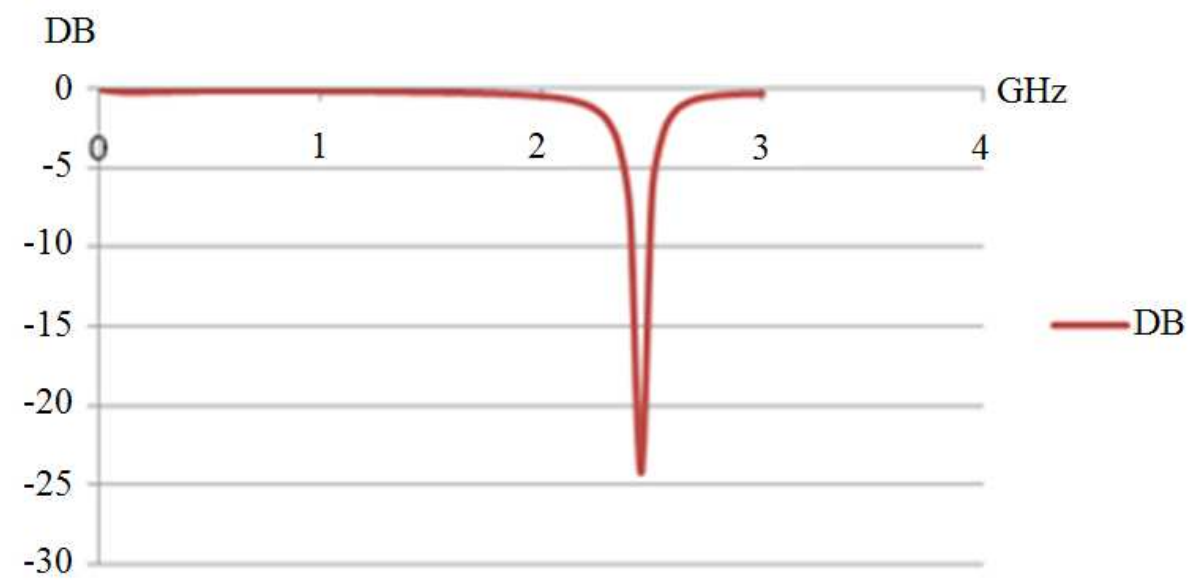

Fig. 3. Microstrip antenna resonant frequency at $2.45 \mathrm{GHz}$

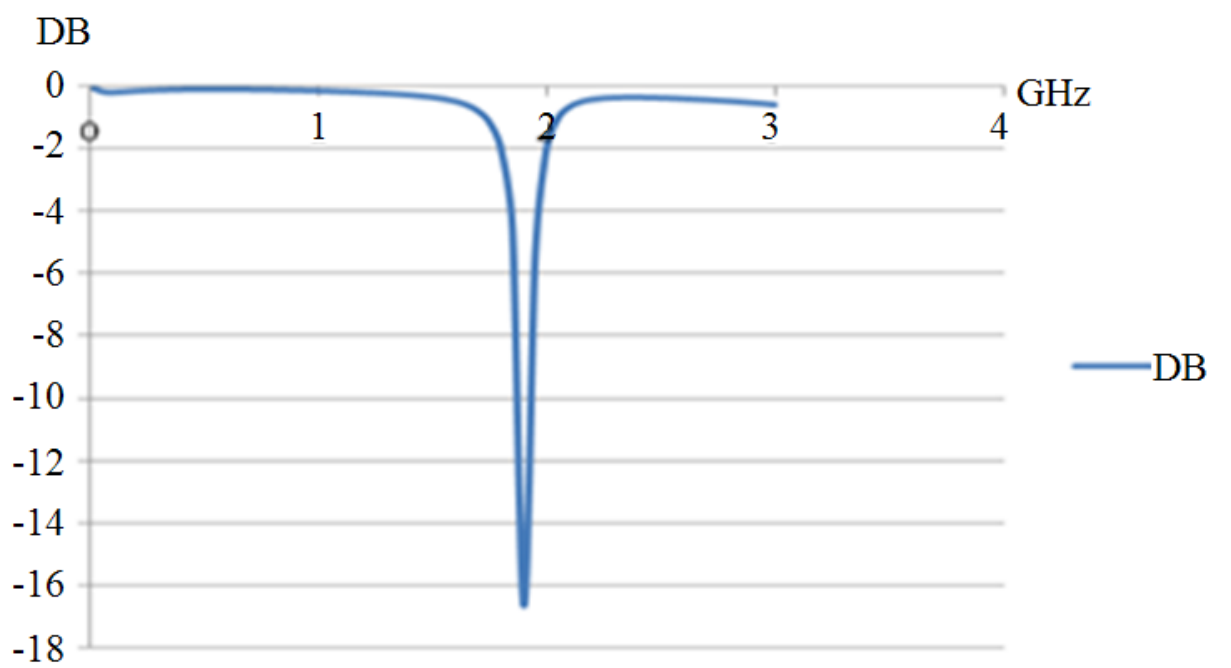

Fig. 4. Microstrip antenna resonant frequency at $1.9 \mathrm{GHz}$

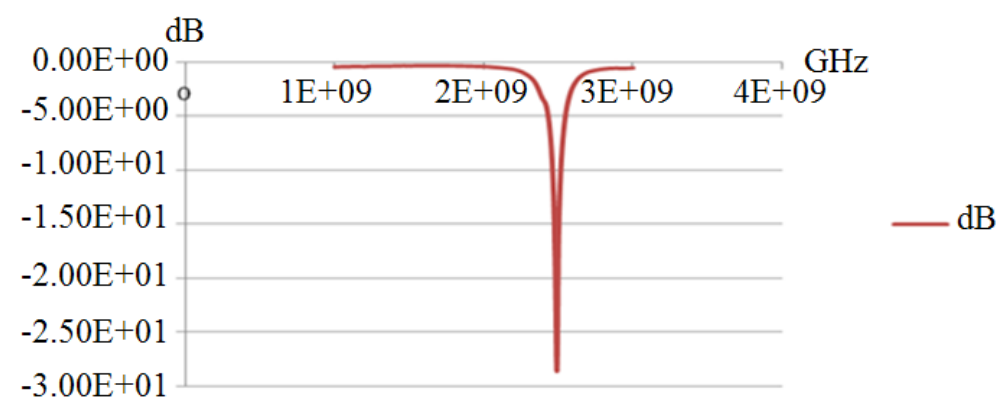

Fig. 5. Transmitter antenna with operating frequency $2.46 \mathrm{GHz}-2.51 \mathrm{GHz}$ 
Leong Kah Meng et al. / American Journal of Applied Sciences 11 (2): 195-201, 2014

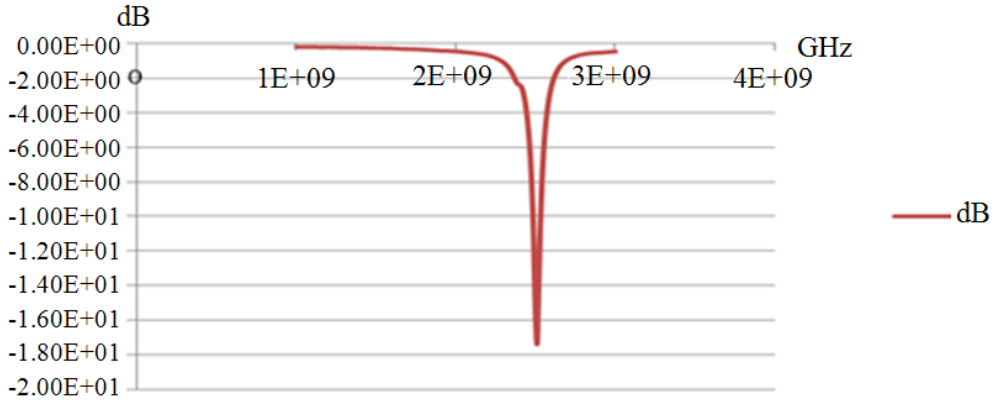

Fig. 6. Receiver antenna with operating frequency $2.48 \mathrm{GHz}-2.53 \mathrm{GHz}$

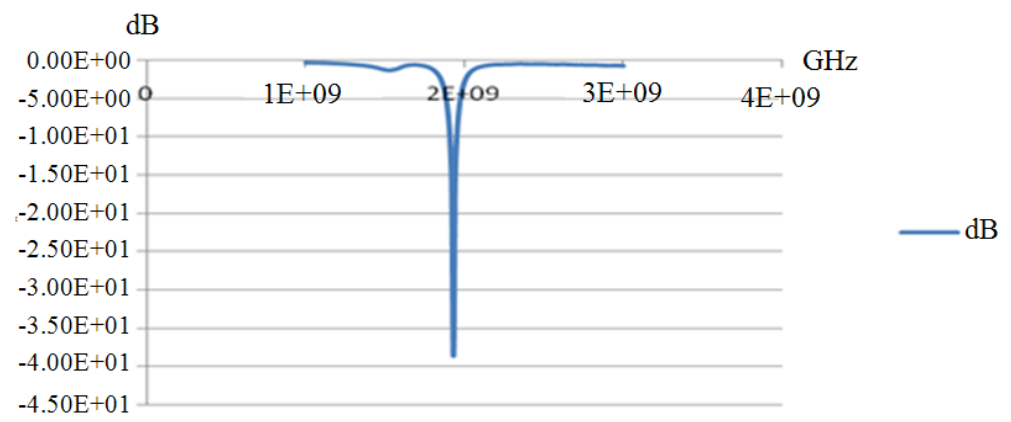

Fig. 7. Transmitter antenna with operating frequency $1.91 \mathrm{GHz}-1.95 \mathrm{GHz}$

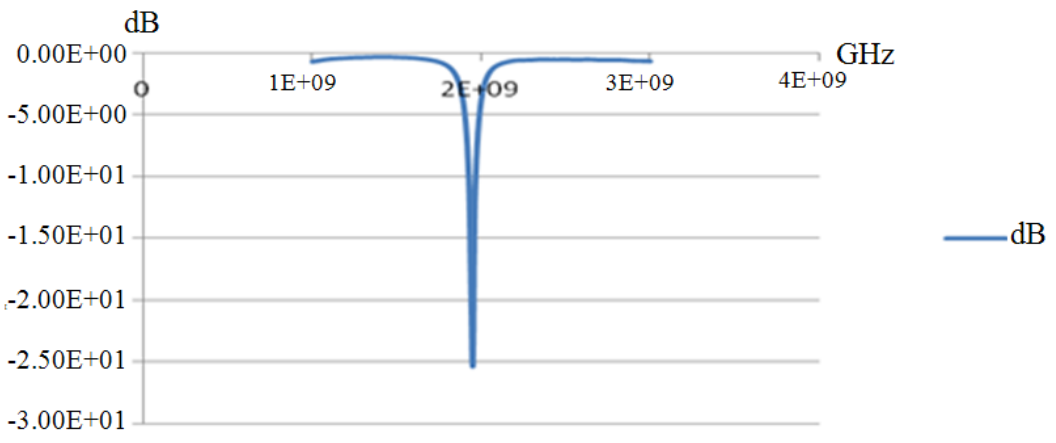

Fig. 8. Receiver antenna with operating frequency $2.46 \mathrm{GHz}-2.51 \mathrm{GHz}$

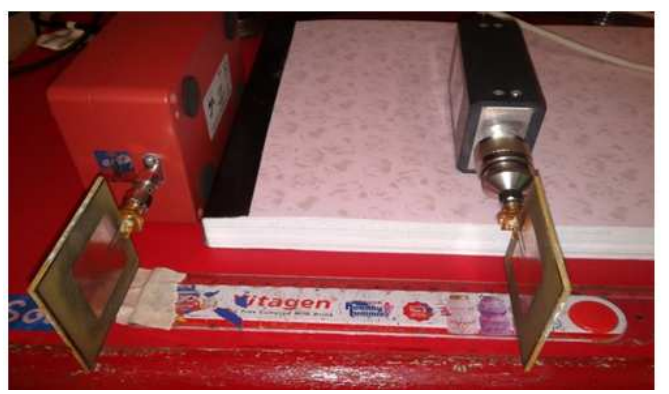

Fig. 9. Measure for power received from $3 \mathrm{~cm}$ to $30 \mathrm{~cm}$ 


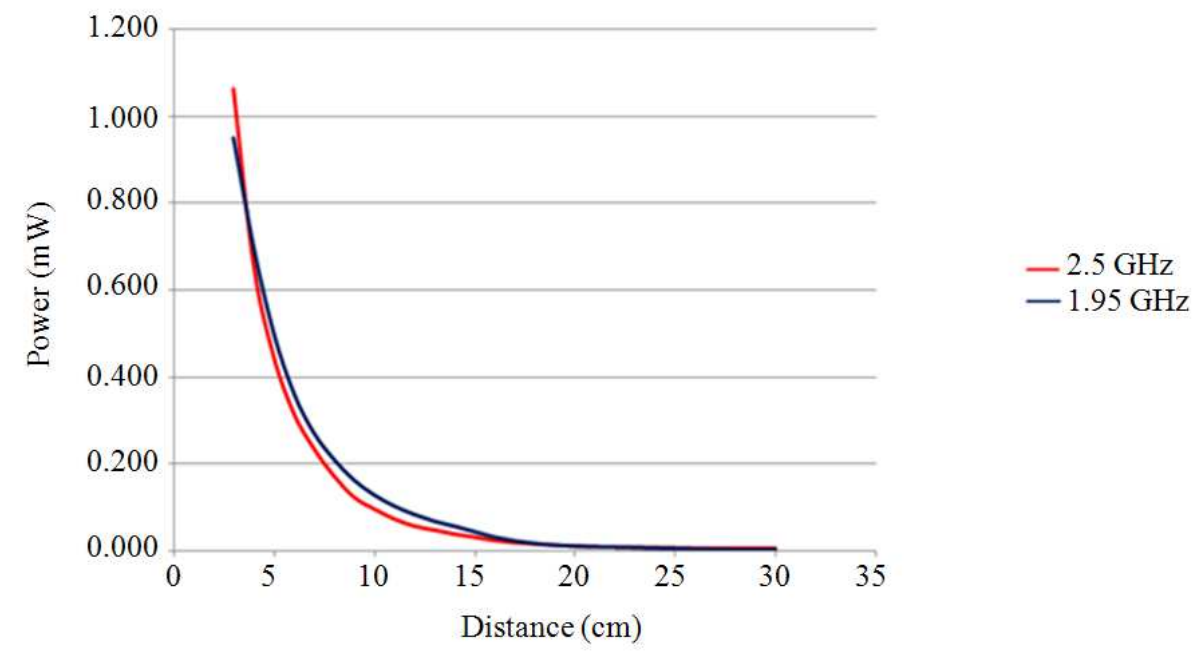

Fig. 10. Comparison power received between two different frequencies $(1.94 \mathrm{GHz}$ and $2.5 \mathrm{GHz})$

This Vaunix Lab Bricks can generate from $1.6 \mathrm{GHz}$ up to $20 \mathrm{GHz}$ and the able to generate maximum $10 \mathrm{~mW}$ of power. The signal generator consist of a SMA connector which can be feed with the connection of microstrip antenna which using the same SMA connector. It was set to generate the frequency as measured by using network analyzer for the antenna pairs. Frequency of $1.94 \mathrm{GHz}$ was used on lower frequency antenna pairs and $2.5 \mathrm{GHz}$ was used for another. Maximum power of $10 \mathrm{~mW}$ was used for transmission and its received power was measured by the USB power meter and displayed on computer screen. Figure 9 shows the experiment set up for the measurement.

\section{RESULTS}

From the Fig. 10 above, we can clearly see that with the increment in the distance, the power receive by the microstrip antenna is decreased exponentially. When the distance increase from $3 \mathrm{~cm}$ to $4 \mathrm{~cm}$, the energy receive by using $2.5 \mathrm{GHz}$ resonant frequency is higher than 1.94 $\mathrm{GHz}$ resonant frequency. The highest power received by resonant frequency $2.5 \mathrm{GHz}$ was $1.062 \mathrm{~mW}$ and for power received by resonant frequency $1.94 \mathrm{GHz}$ was $0.95 \mathrm{~mW}$. However, when the distance keep increasing from $5 \mathrm{~cm}$ to $10 \mathrm{~cm}$, the resonant frequency $1.94 \mathrm{GHz}$ was carry higher power compare with resonant frequency $2.5 \mathrm{GHz}$. The energy received by resonant frequency $2.5 \mathrm{GHz}$ was $0.096 \mathrm{~mW}$ and energy received by resonant frequency $1.94 \mathrm{GHz}$ was $0.131 \mathrm{~mW}$. The power received by the microstrip antenna with resonant frequency $2.5 \mathrm{GHz}$ and $1.94 \mathrm{GHz}$ approximately equal to zero after $25 \mathrm{~cm}$ and $27 \mathrm{~cm}$ respectively.

\section{DISCUSSION}

This part will discuss about the relation between the power received by each antenna pair and its relation on distance increment. Relation of power versus distance was plotted as shown in Fig. 10.

From the result we can seen that resonant frequency of $2.5 \mathrm{GHz}$ had a better performance than resonant frequency of $1.9 \mathrm{GHz}$ in a shorter distance. However, with the increasing distance resonant freqency of 2.5 $\mathrm{GHz}$ shows a large among of power loss in the radiation process. In the other hand, the power received from resonant frequency of $1.9 \mathrm{GHz}$ is higher compare to resonant frequency of $2.51 \mathrm{GHz}$ when the distance increase. From the comparison above, we can saw that the lower resonant frequency $1.94 \mathrm{GHz}$ was able to travel for a longer distance compare with resonant frequency at $2.5 \mathrm{GHz}$.

In fact, the signal will not travel in straight line of sight. Instead, it will deflect while the distance is further from the transmitter. If the receiver is moved by following the path of the signal travels, then the energy received will be much higher. Some of the reasons that can affect this wireless energy transfer for example noise and other frequency source might highly affect the performance of this microstrip antenna pair. By increase the transmitting energy and carry out the experiment in a room with anechoic chambers can help to improve the efficiency of the system performance. Anechoic chambers can help suppress any reflections that could disturb the measurements of antennas. 
The system only showing a maximum power efficiency of just $10 \%$ for the very prototyping setup. There are several factors cause the low performance of system. First is the frequency deviation during fabrication process. The frequency mismatch problem can be reduced by proper reevaluate the resonant frequency of the microstrip antenna. Proper tuning of the resonant frequency does play an important role in maintaining the high efficiency of the overall system. Another factor will be the impedance mismatch of the antennas pairs with the circuitry. Maximum power transfer is guaranteed if there is a match in both impedance of the circuit and the antennas. It is very vital to maximize the power transfer due to the very limited power available for the system. The mismatch of the impedance causes lower power transferred and received. Proper design of the circuitry to match the impedance of the antenna will be our future design consideration.

\section{CONCLUSION}

The project did provide the fundamental idea on implementing the wireless power transfer charging system by using microstrip antenna as transmitter and receiver. The performance of this system was measured and being discussed. The characteristic of microstrip antenna has been study. The larger the size of microstrip antenna, the lower the resonance frequency which microstrip antenna operate with and vice versa. Microstrip antenna with resonant frequency $1.94 \mathrm{GHz}$ and $2.5 \mathrm{GHz}$ were fabricated. Approximately of two percent of deviation resonant frequency occur during fabrication of microstrip antenna. Lower resonant frequency, $1.94 \mathrm{GHz}$ can travel longer distance compare to higher resonant frequency, $2.5 \mathrm{GHz}$. The system exhibit an efficiency of about $10 \%$. Frequency detune and impedance mismatch is a serious problem which cause the inefficient of the performance of microstrip antenna. More thorough research and improvement will be done in order to make the system possible to be implemented in supplying energy to the electronic devices.

\section{ACKNOWLEDGEMENTS}

This research is a collaboration between Universiti Teknologi Malaysia (UTM) and Institut Jantung Negara (IJN). The authors gratefully acknowledge the research grant provided to this study by Research Management Centre sponsored by Ministry of Higher Education, Malaysia. Vot: 04H41 (Reseach University Grant), Universiti Teknologi Malaysia, Johor Bahru, Malaysia

\section{REFERENCES}

Casanova, J.J., Z.N. Low, J. Lin and R. Tseng, 2009. Transmitting coil achieving uniform magnetic field distribution for planar wireless power transfer system. Proceedings of the IEEE Radio and Wireless Symposium, Jan 18-22, IEEE Xplore Press, San Diego, CA., pp: 530-533. DOI: 10.1109/RWS.2009.4957405

Chang, S.H. and W.J. Liao, 2010. A franklin array antenna for wireless charging applications. Progress Electromagnet. Res. Symp. Proc., 2: 279-283.

Goh, C.K., X. Qing, Z.N. Chen and T.S.P. See, 2012. Effect of wireless charging antennas on transmission of an antenna pair through human body. Proceeding of the IEEE Asia-Pacific Conference Antennas Propagation, Aug. 27-29, IEEE Xplore Press, Singapore, pp: 57-58. DOI: 10.1109/APCAP.2012.6333138

Heikkinen, J. and M. Kivikoski, 2001. Performance and efficiency of planar rectennas for short-range wireless power transfer at $2.45 \mathrm{GHz}$. Microwave Optical Technol. Lett., 31: 86-91. DOI: 10.1002/mop. 1365

Lin, J., 2011. Wireless energy transfer and conversion IEEE Microwave Mag., 12: 126-139. DOI: 10.1109/MMM.2011.941422

Peng, L., J. Wang, H. University, C.L. Hangzhou and M. Asger et al., 2011. Performance analysis and experimental verification of mid-range wireless energy transfer through non-resonant magnetic coupling. J. Electromagnet. Waves Applic., 25: 845855. DOI: $10.1163 / 156939311794827186$

Sim, Z.W., R. Shuttleworth, M.J. Alexander and B.D. Grieve, 2010. Compact patch antenna design for outdoor RF energy harvesting in wireless sensors networks. Progress Electromagnet. Res. Pier, 105: 273-294. DOI: 10.2528/PIER10052509

Yoon, I.J. and H. Ling, 2012. Realizing efficient wireless power transfer in the near-field region using electrically small antennas. Wireless Power Transfer-Principles Eng. Exp. DOI: 10.5772/28897

Yu, X.F., S. Sandhu, S. Beiker, R. Sassoon and S. Fan, 2011. Wireless energy transfer with the presence of metallic planes. Applied Phys. Lett., 99: $214102-$ 214102. DOI: $10.1063 / 1.3663576$ 